\title{
Mathematically Reduced Chemical Reaction Mechanism Using Neural Networks
}

\author{
Final Report
}

September 22, 2003 through August 31, 2007

\author{
Prepared by \\ Ziaul Huque \\ Department of Mechanical Engineering \\ (For Nelson Butuk) \\ Principal Investigator \\ Department of Mathematics \\ Prairie View A \& M University \\ Prairie View, Texas 77446-4189
}

March 24, 2008

DOE Grant Number: DEFG26-03NT-41913

Office of Sponsored Programs

Prairie View A \& M University:

P. 0. Box 667

Prairie View Texas 77446-066? 


\title{
Disclaimer
}

*This report was prepared as an account of work sponsored by an agency of the United States Government. Neither the United States Government nor any agency thereof, nor any of their emfiloyees, makes any warranty, express or implied, or assumes any legal liability or responsibility for the accuracy, completeness, or usefulness of any information, apparatus, proluct, or process disclosed, or represents that its use would not infringe privately owned rights. Reference herein to any specific commercial product, process, or service by trade nante, trademark, manufacturer, or otherwise does not necessarily constitute or imply its endorsement, recommendation, or favoring by the United States Government or any agency thereof. The views and opinions of authors expressed herein do not necessarily state or reflect those of the United States Government or any agency thereof**

\begin{abstract}
This is the final technical report for the project titled "Mathematically Reduced Cheraical Reaction Mechanism Using Neural Networks." The aim of the project was to develop an efficient chemistry model for combustion simulations. The reduced chemistry molel was developed mathematically without the need of having extensive knowledge of the chemistry involved. To aid in the development of the model, Neural Networks (NN) was used via a new network topology know as Non-linear Principal Components Analysis (NPCA).

A commonly used Multilayer Perceptron Neural Network (MLP-NN) was modified to implement NPCA-NN. The training rate of NPCA-NN was improved with the Generalized Regression Neural Network (GRNN) based on kernel smoothing techniques. Kernel smcothing provides a simple way of finding structure in data set without the imposition of a parkmetric model. The trajectory data of the reaction mechanism was generated based on the optimization techniques of genetic algorithm $(\mathrm{GA})$. The NICA-NN algorithm was then used for the reduction of Dimethyl Ether (DME) mechanism. DME is a recently discovered fuel mace from natural gas, (and other feedstock such as coal, biomass, and urban wastes) which can be used in compression ignition engines as a substitute for diesel. An in-house twodimensional Computational Fluid Dynamics (CFD) code was developed based on Meshfree techrique and time marching solution algorithm. The project also provided valuable research expintence to two graduate students.
\end{abstract}




\section{TABLE CONTENTS}

Dischimer

Abstract

1.0. Introduction 4

2.0 Background Information 5

2.1 Neural Networks (NN 5

2.2 Nonlinear Principal Component Analysis (NPCA-NN) 6

2.3 Improving the training rate of NPCA-NN 7

2.3.1 Generalized Regression Neural Network (GRNN) 8

3.0 Generation of Trajectory Data 13

4.0 Application of NPCA-NN Algorithm to the Reduction of Dimethyl Ether (DME) Mechanism.

5.0 CFD Code Development and Implementation

5.1 Meshfree Techniques $\quad 22$

5.2 Grids 23

5.3 Boundary Conditions $\quad 24$

5.4 Kernel Methods 26

5.5 Complex Variables Method for Obtaining Derivatives _ $\quad 27$

5.6 Runge-Kutta Method (RKM) 28

5.7 The Euler Equations of flow in two-dimensional form:

5.8 The Euler Equations of flow in two-dimensional form:

5.9 Solving the ODEs Using the RKM 33

5.10 Comparison with Lobo Solver 33

6.0 Conclusions $\quad 35$

7.0 References $\quad 35$ 


\section{Mathematically Reduced Chemical Reaction Mechanism Using Neural Networks}

\subsection{Introduction}

In combustion simulations, one frequently encounters chemical mechanisms consisting of micre than 500 species participating in more than 2000 chemical elementary reactions. To modal this mechanism with such a large number of species is computationally prohibitive. Hence, it is desired to reduce the number of species to be modeled to a number that is suitable for CFD computations, and yet be able to accurately represent the full detailed meclianism. These species are modeled by very high-dimensional systems of non-linear differential equations which exist as isolated regions in phase-space, and may geometrically exhibit shapes such as circles, tori or other manifolds [Kirby and Miranda, 1999]. It is desirable to approximate these isolated regions of the phase-space by a mathematical model of lower dimension than the dimension of the original ambient space. Several techniques have been used to reduce the reaction mechanism that describes the chemical transformations of a given reaction. Mathematical techniques used in mechanism reductions have been desctibed by Tomlin et al. (1997). The methods used can be broadly classified as those based on response surface modeling, and various techniques based on approximate reductions. Recintly introduced approximate methods are those based on the analysis of time-scales of the reaction set. These are the Computational Singular Perturbation (CSP) method [Lam and Goussis, 1988], the method of Intrinsic Low-Dimensional Manifold (ILDM) [Maas and Pope, 1992], and the newly introduced Low Dimensional Center Manifold (LDCM) [Butuk and Pemba, 2001]. The current work is based on another new technique that appears to be pronising in terms of efficiency and ease of implementation (Butuk, 2001). The technique is known as Nonlinear Principal Component Analysis-Neural Network (NPCA-NN) and uses Neural Network (NN). The method is entirely mathematical and does not require any extensive knowledge of the chemistry involved.

The NPCA-NN constructs a global transformation mapping that maps data from a high dimensional state space to a low dimensional space. Let $x_{1}, x_{2} \ldots x_{n}$ be a set of yariables (or coordinates-for a given mechanism, the variables are the different species) of an $n$ dimmsional state space. In practice, it has been shown that most of the n-dimensional state space is redundant with most of the data occupying only a small region of the space. If we assume that the variables can be generated by a few $\mathrm{m}(\mathrm{m}<\mathrm{n})$ underlying variables $\theta \in R^{m}$, then $x_{1}=f_{1}\left(\theta_{1}, \theta_{2} \ldots \theta_{m}\right), x_{2}=f_{2}\left(\theta_{1}, \theta_{2} \ldots \theta_{m}\right), \ldots x_{n}=f_{n}\left(\theta_{1}, \theta_{2}, \ldots \theta_{m}\right)$, where $f_{1}(f)$ fn $($.$) are$ non-linear continuous functions $\mathrm{R}^{\mathrm{n}} \rightarrow \mathrm{R}$. Although the dimensionality of the data vector $\mathrm{x}$ is $n$, this number ignores the relation between the coordinates. The n coordinates are so closely related that there is really just $m$ degrees of freedom, namely the hidden variables $\theta$. We say that the intrinsic dimensionality of the data is $\mathrm{m}$. It is the purpose of NPCA to construct the mapping procedure from $x$ to $\theta$. It has been shown that $\mathrm{NN}$ can provide a very powerful tool for construction of this reduction mapping. Butuk et. al, 2001, applied this procedure for the first time to the dimensionality reduction problem of a representative chemically reactive system describing complex combustion processes.

The major objectives of the current work are as follows:

1. Improve the training rate of the NPCA-NN algorithm developed previously, 
2. Develop a method to determine optimum trajectory data of reaction mechanisms needed to use NPCA-NN.

3. Apply the NPCA-NN algorithm to the reduction of Dimethyl ether (DME) mechanism.

4. Couple the developed NPCA-NN model to the KIVA CFD code.

5. Test the CFD code on a few other simple sample mechanisms

6. Student Education in Computational Applied Mathematics

\subsection{Background Information}

2.1 Neural Networks (NN)

Extensive literature on NN exists and a good introductory text is the book by Bharath and Drosen, (1994). The basic element of a neural network is called, naturally, a neuron. It is also known as a node (or unit), processing element, or perceptron (upper schematic of Figure 1). The links between neurons are called synapses weights. The input signal to a given neuron is calculated as follows: The outputs of the preceding neurons of the network (i.e., the $X_{1}, X_{2}, X_{3}$ in Figure 1) are multiplied by their respective synapse weights, $P_{1}, P_{2}$, and $P_{3}$. These results are added resulting in the value $t$ that is delivered to the given neuron. The state or activation value of this neuron is calculated by the application of a threshold function to its input value, resulting in the final value $Y$. This threshold function, also called activation function, is frequently nonlinear and must be chosen carefully, as the performance of the neural network depends heavily on it.

Figure 1 (lower schematic) shows the commonly used Multilayer Perceptron Neural Network (MLP-NN). This type of network shows data flow in one direction (i.e., it is a unidirectional network). Its simplicity and stability make it a natural choice for applications suct as data analysis, classification, and interpolation. Consequently, it is particularly suitible for physical modeling, and, in fact, there are many real-world applications of this type of network. A fundamental characteristic of this network is the arrangement of neurons in lityers; such as, there must be at least two layers; data input and data output. As the performance of two-layer Neural Networks is very limited, it generally includes at least one mor intermediate layer, also called the hidden layer. Each neuron is linked to all of the neurons of the neighboring layers, but there are no links between neurons of the same layer. The behavior of the MLP network is static; its output is a reflection of its respective input. It must be previously trained using real data in order to perform adequately.

During the training step, real data (input and output) will be continuously presented to the NN. It periodically compares real data with results calculated by the neuron network. The diffirence between real and calculated results (i.e., the error) is processed through a relatively easy mathematical procedure (known as backpropagation (BP) training), which adjusts the value of the synapse weights in order to minimize this error. This is an important feature of the Neural Networks; their knowledge is stored in their synapse weights. Next we describe how the MLP network was modified to implement NPCA-NN. 


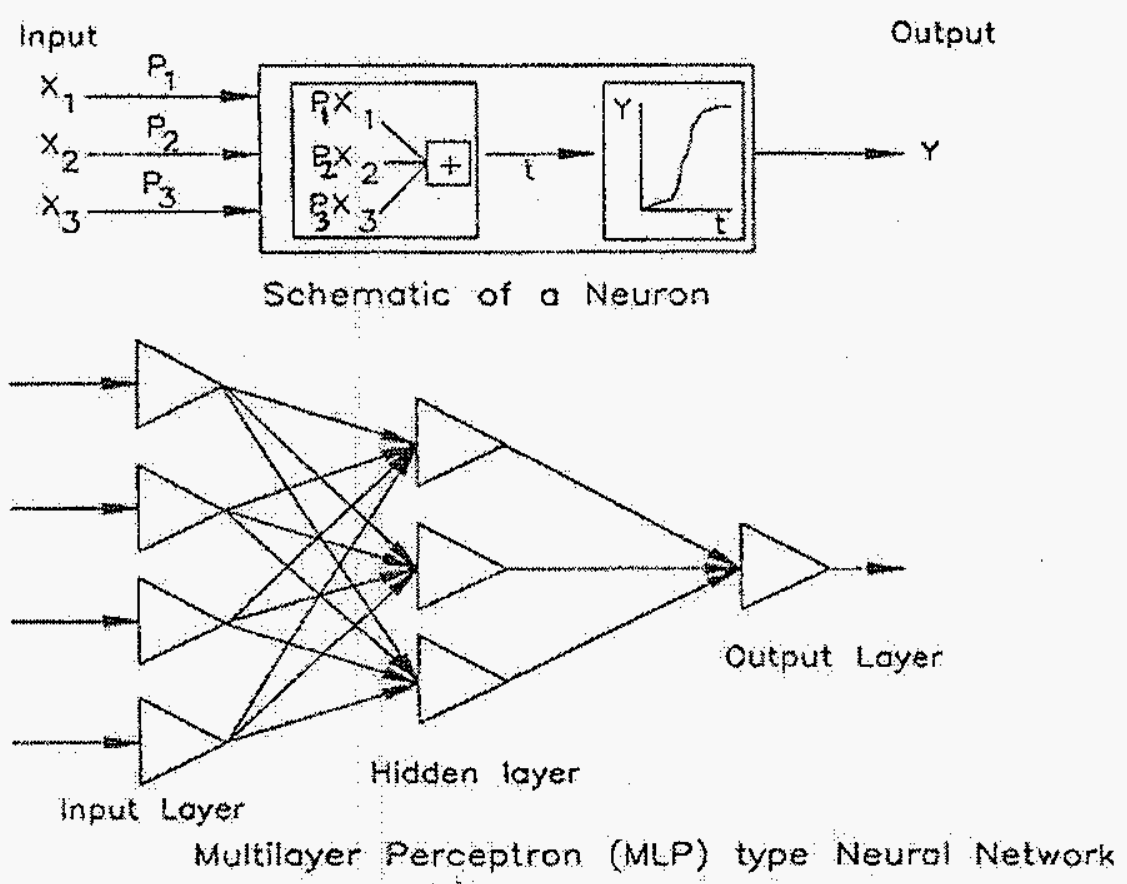

Figure 1. Neural Network Model

\subsection{Nonlinear Principal Component Analysis (NPCA-NN)}

The dimensionality reduction technique of Non-linear Principal Component Analysis (NPCA-NN) [Kramer, 1991 and Kirby and Miranda, 1999] is an empirical (i.e requires data to implement) procedure. As indicated above, it is the purpose of NPCA-NN to construct the mapping procedure from $\mathrm{x}$ to $\theta$. It has been shown that $\mathrm{NN}$ can provide a very powerful tool for construction of this reduction mapping.

in brief, the NPCA-NN method involves determination of a mapping

$$
\mathrm{G} * \mathrm{x} \rightarrow \theta
$$

Fron $R^{n}$ to $R^{m}$ where $m<n$. It will also be desired to implement the inverse mapping from $R^{m}$ to $R^{2}$, i.e.,

\section{$\mathrm{H}: \theta \rightarrow \mathrm{x}$}

In essence a composite identity mapping is constructed

\section{$\mathrm{HoG} x \rightarrow \mathrm{x}$}

$\mathrm{NN}$ are constructed to implement this composite mapping, $H \circ \mathrm{G}$. Given sufficient coordinates $\mathrm{x}$ (corresponding to a large number of trajectories) of the n-dimensional state space, NN will construct reduced dimensional coordinates globally. To construct this mapping the NN requires extensive input data $\mathbf{x}(\mathbf{t})$. In chemical kinetics, it is usually computationally expensive to produce the required training data $x(t)$ for all trajectories of the 
reaction state space. In this study we have developed a procedure to address this short coming by using the techniques of evolutionary computations. This procedure for generating representative trajectories for NPCA-NN will be outlined in details below.

The MLP-NN was modified to implement the NPCA-NN. Essentially the NPCA-NN, consists of two standards MLP-NN connected together in series (See Figure 2). The first netvork implements the mapping $G$. Here a sample trajectory. $x(t)$ of the original $n-$ diminsional system is given to the input layer, it is then mapped onto the mapping layer (hidilen layer of lst network) before being compressed into an m-dimensional bottleneck laye or trajectory $9(t)$. The second network is for reconstruction via the mapping $H$. The trajectory $9(\mathrm{t})$ is used for reconstruction through a demapping layer into the output outer layes, where the original trajectory $x(t)$ is reproduced. Note that the dimension of the reduced reacion state space $m$ is simply changed by changing the number of neurons in the bottleneck layer of Figure 2 . This ease of changing the dimension of the reduced space makes this method quite flexible. .

Therefore, NPCA-NN is build from the standard Multi- layered Perceptron (MLP-NN), and is trained to implement the identity mapping $\mathrm{x} \sim \mathrm{x}$. The weights in this network are detemined such that the mean square error is as small as possible, i.e.

$<\mathrm{II} \% \cdot(\mathrm{H} 0 \mathrm{G})(\mathrm{x}) 1 \mathrm{r}>=$ minimum where 11.11 is the norm to be specified.

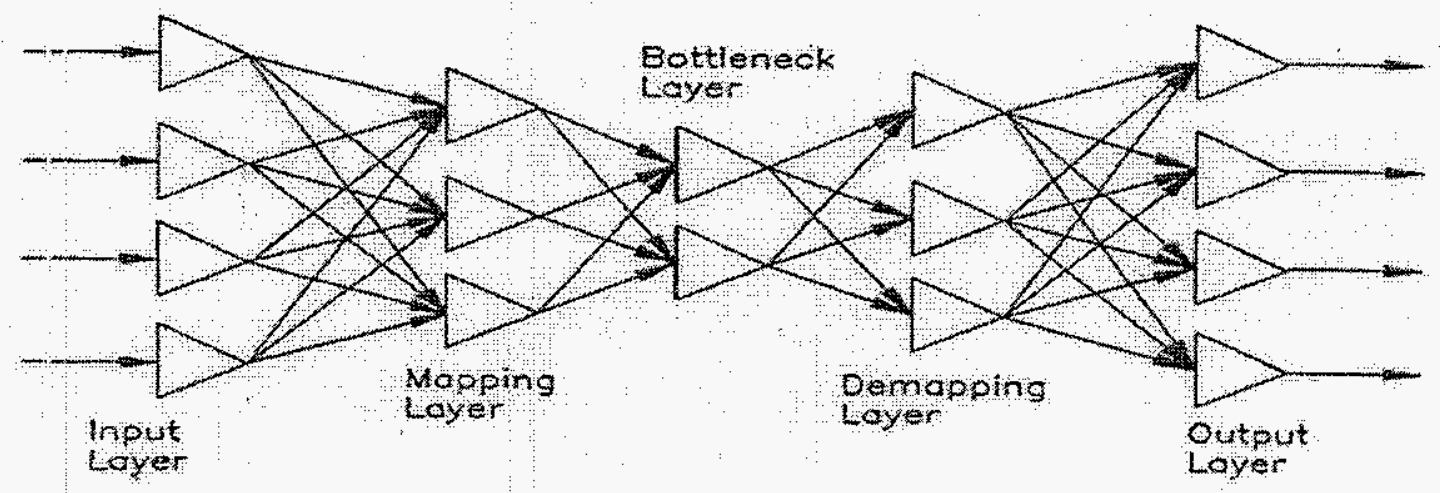

Figure 2. Non-Linear Principal Component Analysts Neural Network

\subsection{Improving the training rate of NPCA-NN}

The NPCA is a NN topology with five layers, in which the input layer has the same number of nodes as the output layer. This allows the input values to be used as target output values of the network during training. The first part of the network approximates the mayting $G$. It contains the mapping layer. The middle layer is the bottle-neck layer consisting of the $\mathrm{m}$ nodes (i.e. desired reduced dimension $\mathrm{m}$ ). The last part of the network implements the mapping $\mathrm{H}$ and contains the de-mapping layer. This layer takes the output of the middle layer and maps it onto the output layer. It is on the bottle-neck layer, that the reduced manifold of the chemistry is reconstructed. 
Among the different implementation of Neural Networks (NN), the network structure that posses the fastest training time, is the Generalized Regression Neural Networks (GRNN). Below is given a brief description of GRNN, present results of actual implementation on a test problem and highlight some of its advantages.

\subsubsection{Generalized Regression Neural Network (GRNN)}

The mathematical background for GRNN is based on the statistical kernel smoothing techriques. Kernel smoothing provides a simple way of finding structure in data set without the irrposition of a parametric model (i.e., models based on basic functions such as logs, exponents, sines, and cosines). This smoothing without using known models is known as non-parametric regression.

Consider a parametric regression in which you want to fit data to a linear function. Giver a data set

$$
\left\{\left(X_{i}, Y_{i}\right\}_{i \neq 1}^{n}\right.
$$

The linear fit to the data is of the form:

$$
Y_{i}=\beta_{o}+\beta_{1} X_{i}+\varepsilon_{i}
$$

$\beta_{0}$ ard $\beta_{1}$ are model parameters determined from data using appropriate numerical fitting techniques. $\varepsilon_{i}$ are the errors of the approximation assumed to be symmetric random variables havitig mean zero.

Call the developed approximating function $\mathrm{m}(\mathrm{X})$. The function $\mathrm{m}$ for which

$$
E\{Y-m(X)\}^{2}
$$

is minimized, is the conditional mean $Y$ given $X$ :

$$
m(X)=E(Y \mid X)
$$

in nen-linear regression theory, $\mathrm{E}(\mathrm{Y}] \mathrm{X})$, the expected value of $\mathrm{Y}$ given $\mathrm{X}$ is:

$$
E[Y \mid X]=\frac{\int_{-\infty}^{\infty} X f(X, Y) d Y}{\int_{-\infty}^{\infty} f(X, Y) d Y}
$$

whese $f(X, Y)$ is the joint probability density function (pdf) of $X$ and $Y$.

Now if we remove the restriction that $\mathrm{m}$ belongs to a particular family of models (i.e. let the data speak for themselves) then you now have what is known as non-parametric regression. The kemel smoothing approach, described later, of GRNN is based on "Parzen's Metood of Density Estimation" first proposed by Parzen in 1962. 
The method to be described below is a way of estimating the pdf, $f(X, Y)$ above, given only a saraple data set $\left\{\left(X_{i}, Y_{i}\right\}_{i=1}^{n}\right.$.

Suppose we have an unknown sample whose value of $\mathrm{X}$ variable is $\mathrm{X}$. We want to know the estimate $y$ of $Y$ given this value of $X$. Compute:

$$
\begin{gathered}
D_{i}^{2}=\left(x-X_{i}\right)^{T}\left(x-X_{i}\right) \\
h_{i}=\exp \left[\frac{-D_{i}^{2}}{2 \sigma^{2}}\right] \quad i=1 . . n
\end{gathered}
$$

Several methods of choosing $\sigma$ are available and can be different for each data point $X_{i}$. I this work we have used a constant $\sigma$.

The approximation for $y$ given $x$ will now be given by:

$$
y=\frac{\sum_{i=1}^{m} h_{i} Y_{i}}{\sum_{j=1}^{n} h_{i}}
$$

GRNN, implements the above theory in a Neural Network, which consist of four layers (Figure 3). The first layer is the input layer, which is fully connected to the next layer and is just tsed to distribute the input vector to each node in the next layer. The second layer is the first hidden layer (also called Pattem Layer). This layer consists of $N$ Processing elements or nodes, where $\mathrm{N}$ is the number of samples within a training data set and each node represents the input yector, $\mathrm{X}_{\mathrm{j}}$, associates with the $\mathrm{j}$-th sample (or observation) in training data. In each nods, each input vector is stbtracted from the vector assigned to the node, $X_{j}$. This difference is then squared by the node. The result is fed into a non-linear Kernel, which is usually an exponential function.

The choice of the smoothing parameter of the Kernel, $\sigma$, is important. It has the effect of smoothing the training examples. Small values of $\sigma$ tend to make each training point distinct, whereas large values will force a greater degree of interpolation between the training observations. The output of the Kernel function is a weighted average of the outputs of each trairing observation. The weighting is related to $\sigma$ and the distance of the input vector from $X_{j}$, the node vector. Therefore, points nearby to the node vector contribute most heavily to the overall output of the Kernel. Choosing, $\sigma$, determines the width of an area in the input space to which each node responds.

The third layer, is the $2^{\text {nd }}$ hidden layer (or Summation layer) and consists of only two nodes. The input to the first node, is the sum of the $1^{\text {st }}$ hidden layer outputs (or activations) each weighted by the observed output $y_{j}$ corresponding to $X_{\mathrm{f}}$. In other words, the input is simply a dot product between the $1^{\text {st }}$ hidden layer outputs and the desired network output vecor, $y$. The input to the $2^{\text {nd }}$ node, is the summation of the $1^{\text {st }}$ hidden layer activations. The $1^{\text {st }}$ node represents the numerator of the above equation 5 , and the $2^{\text {nd }}$ node represents the denominator. 


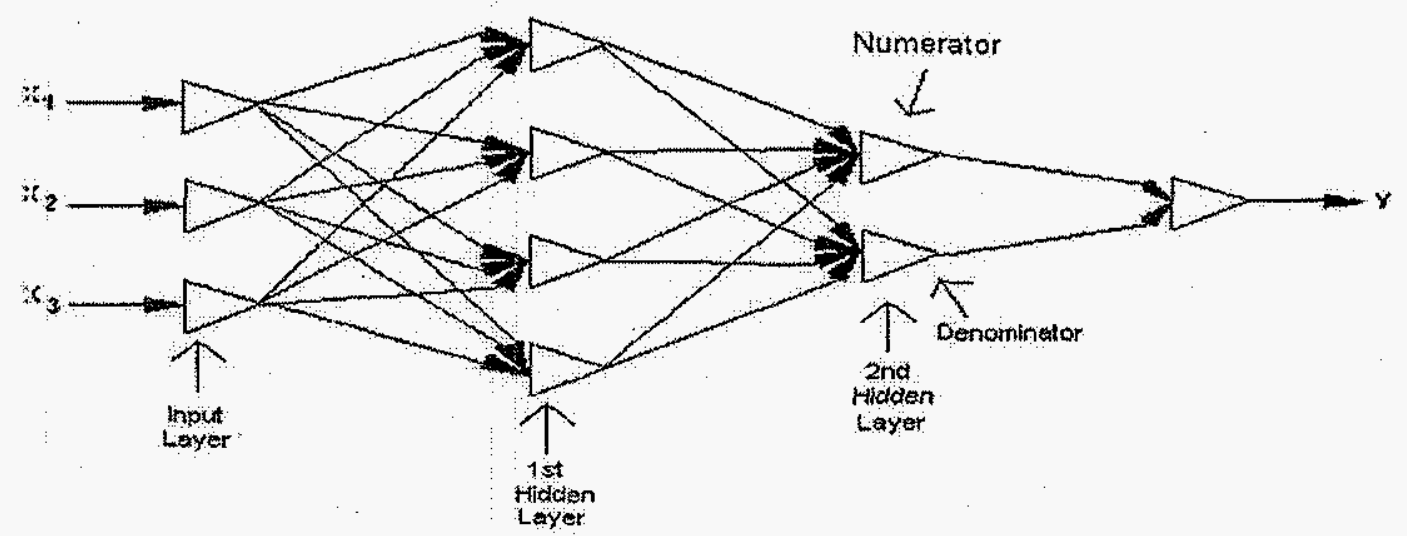

Figure 3. Neural Network Implementation of Generalized Regression

The fourth layer is the output layer. It receives the two outputs from the $2^{\text {nd }}$ hidden layer and livides them according to equation 5 , above to yield an estimate for $y$. Note that the $2^{\text {nd }}$ hidden layer always has exactly one more node, than the output layer. The $2^{\text {nd }}$ hidden-tooutput layer weights are just the target values, $y$, so that the estimated network output, is simply a weighted average of the target values, $y$, of the training observations close to a given input case.

When there is a need of a multidimensional (vector) output, the only change to the network, is to add one additional node to the $2^{\text {nd }}$ hidden layer, plus an additional node in the output layer for each element of the output vector.

Overall, the GRNN has only a single parameter, $\sigma$, that needs to be determined during calit ration (training), and therefore can be trained fast. $\sigma$ is optimized during training of the network. Available data is split into two sets-training and test sets. Cross-yalidation with the test set is then used to determine the optimum value of $\sigma$. Alternatively, $\sigma$, can be computed direstly from data. For each $1^{\text {st }}$ hidden layer node, find the RMS distance between its vector, $\mathrm{X}_{\mathrm{j}}$, and the center of its $\mathrm{M}$, nearest neighbors. Assign this value to $\sigma_{\mathrm{j}}$. Then assign the average of all $\sigma_{\mathrm{j}}$ 's to $\sigma$. Another characteristic of GRNN, which makes it fast in training, is that there is only the linear output layer beyond the $1^{\text {st }}$ hidden layer, this guarantees that the network will converge to a global minimum. The linearity is with respect to the weights of the network and not the input variables $\mathrm{x}$. Finally for large scale problems the GRNN can be efficently parallelized and implemented in a super computer with many computing nodes. This is because the GRNN consists of a large number of simple processes that can be exeruted mostly in parallel.

The project aim as indicated above is to develop an algorithm for dimension reduction of ctemical systems. The problem may be posed mathematically as 


$$
\begin{aligned}
& f_{1}\left(x_{1} \ldots x_{n}\right)=y_{1} \\
& f_{2}\left(x_{1} \ldots x_{n}\right)=y_{2} \\
& f_{n}\left(x_{1} \ldots x_{n}\right)=y_{n}
\end{aligned}
$$

If we consider only $f_{i}$, then $x \in R^{\mathfrak{n}}$ and $y \in R$. The dimension of the problem in this case is $n$, which can be quite large for most chemical systems. The dimension reduction problem is to reduce the dimension of the input variables from $n$ to $m$, where $m$ is much smaller than $\mathrm{n}$. If the reduced variables are represented $a s \in \mathrm{R}^{\mathrm{m}}$ then our problem can be modeled in terms of $z$ as

$$
g\left(z_{1} \cdots z_{m}\right)=\hat{y}
$$

The objective then is to be able to use GRNN to determine $z$ and $g$, so that $\|y-\hat{y}\|$ is minimized. Here we discuss and present results of work accomplished so far.

The first step was to implement and thoroughly understand the GRNN. The algorithm to implement GRNN was written and implemented in MatLab. In order to test the algorithm, we have used the following non-linear function.

$$
f\left(x_{1}, x_{2}, x_{3}\right)=4 x_{1}^{2}+2 x_{2}^{2}+x_{3}^{2}-x_{1} x_{2}+x_{2} x_{3}-5 x_{1}-9 x_{2}+x_{3}
$$

This is 3 dimensional function and the objective is to reduce it to a one and two dimensional function. $\mathrm{A}$ MatLab $\mathrm{m}$-file was written to implement the function, which was then used to automatically generate $N$, observations or data points for $\left(x, x_{2}, x_{3}\right) \in[-10,10]$. The $\mathrm{N}$ observations formed the set of training data. For test data only 20 points were generated again at random in a similar way as the training data. The results for generating 500 and 1500 data points are presented below in Figures 4 and 5 respectively. The MatLab fles are also attached below.

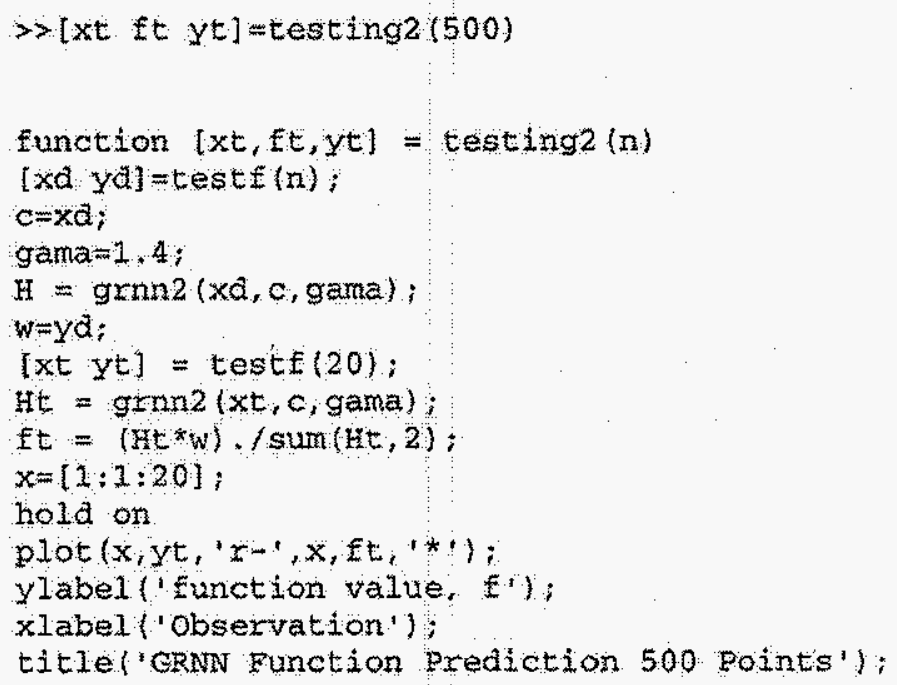


legend ('Actual', 'GRNN Estimate', 1);

hold off

function $[x a ; y]=$ test $($ nd)

* this wil generate na data points $x$ and $y$ for regression analysis * of a particular function

$i d=3$;

$x d=10 * x a n d(1 a, n d)$;

$x a=2^{*} x a-10$;

$x d=\operatorname{sign}(x d){ }^{*} \min (10, a b s(x d))$;

$y=\operatorname{zeros}(n a, 1)$;

$x=\operatorname{zeros}(1,1 \mathrm{a})$

for $j=1$. nd

$x=x d(x, j)$;

$y(1)=4 * x(1)^{\wedge} 2+2 * x(2)^{\wedge} 2+x(3) \wedge 2-x(1) * x(2)+x(2) * x(3)-5 * x(1)$

$9 * x(2)+x(3)$

end.

function $\mathrm{H}=\operatorname{gman} 2(\mathrm{X}, \mathrm{C}, \mathrm{R})$

* Function to imolement Generalized Regression Neural Netwoxk

- It calculates the Natxix of 1st Hiden Layer Activations

$[a, p)=\operatorname{size}(x)$

if $\operatorname{size}(\mathrm{C}, 1) \sim a$

errorfforog ': Dimensions of $\mathrm{C}$ and $\mathrm{X}$ do not Match'l)

end

$m=s i z e(C, 2)$;

- Calculate the Hatrix

$\mathrm{x}=\operatorname{zeros}(\mathrm{p}, \mathrm{m})$;

for $j=1 \mathrm{~m}$ is total nodes in Fidden Layer

$c=c(\div, j) ;$

$A=X-c(:$, ones $(1, p))$;

$Z=\operatorname{sum}(\mathrm{A} \cdot \wedge 2) \cdot\left(\left(2 * \mathrm{R}^{\wedge} 2\right) ;\right.$

$H(\therefore, j)=\exp (-Z)$.

end

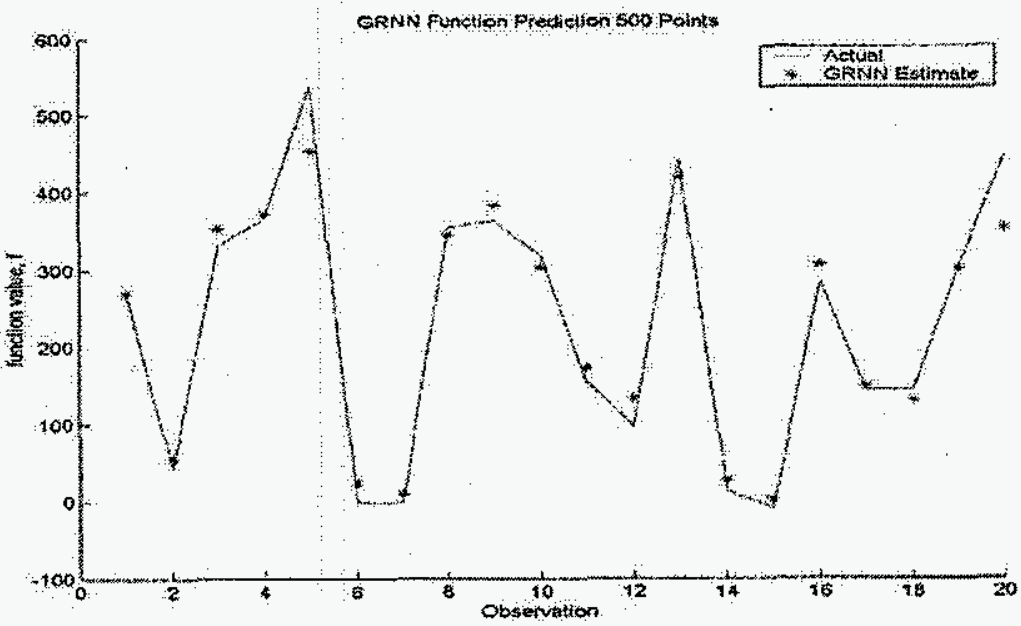

Figure 4 GRNN Results Using 500 Points 


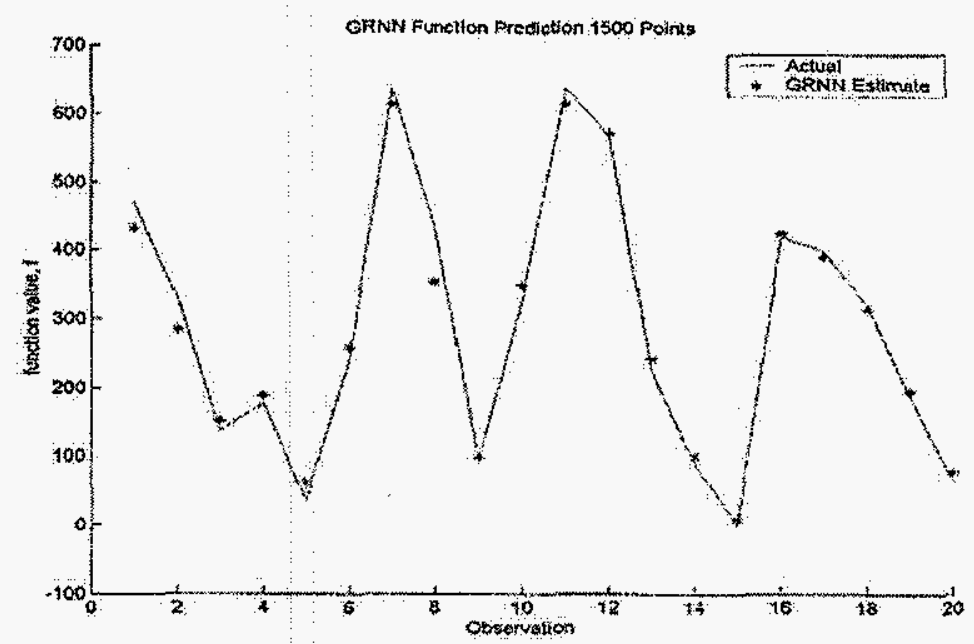

Figure 5 GRNN Results Using 1500 Points

\subsection{Generation of Trajectory Data}

Given sufficient reaction state space variables $x$ (corresponding to a large number of trajpetories) of the $\mathrm{n}$-dimensional reaction state space, NPCA-NN will construct reduced dimensional coordinates globally. As indicated above, NPCA-NN requires data for training. The data required are the trajectory data of the reaction mechanism. A single trajectory of the state space corresponds to one initial condition. To generate many trajectories requires different initial conditions. The trajectories must be a good representation of the state space. We developed an efficient method of generating representative initial conditions to generate the rajectories without using the commercial Design of Experiments (DoE) software.

The method is based on the approach used in initializing a population in the evo utionary optimization technique of Genetic Algorithms $(\mathrm{GA})$. First, the initial species of the problem at one point were encoded into binary bit strings consisting of $n$ pieces of length $I$ such that the total length of the string, $\mathrm{L}=n I$, and each piece encodes the corresponding real-ralued initial species mass fraction at the chosen point $n$ is the number of species present initially. Decoding operates by transferting the bit-string into an integer value between 0 and $i-1$, which is then linearly mapped to the real vector interval [XI, Xu], where $\mathrm{XI}$ is the vector of lower bound of the species concentrations and Xu the vector of upper bound of the conientrations.

Decoding in this manner results in an implicit mapping of the original continuous function space of initial conditions onto grid of sampled points. For a given sample point, a bincry uniform random bit $[0$ or 1$]$ is generated $n I$ times. This procedure can be used to generate any required number of initial data points within the bounds of the state space initial conditions (flammability limits of the fuel). This method is an effective means of choosing a small number of representative samples from all possible combinations of experimental variables. For example, if there are eight variables with three levels each, there would be required 38 or 6,561 experiments to generate all possible combinations. Since this is computationally expensive, our approach is instead used to choose a representative small number from this large number. 


\subsection{Application of NPCA-NN Algorithm to the Reduction of Dimethyl Ether (DME) Mechanism.}

A reduced mechanism for Dimethyl ether (DME) using NPCA-NN was developed. DME is a recently discovered fuel made from natural gas, (and other feedstock such as coal, bionass, and urban wastes) which can be used in compression ignition engines as a substitute for diesel. It produces comparable power to diesel but with cleaner emissions. It has been shown to meet both the California ULEV (ultra-low emission vehicle) standards, as well as the proposed LEV (low emission vehicle) new standards. No catalytic converters are necissary when using this potential fuel. $\mathrm{DME}$, with the chemical formulae $\mathrm{CH}_{3}-\mathrm{O}-\mathrm{CH}_{3}$ is the simplest of the ether compounds. It is a gas at standard temperature and pressure, and a liquid under modest pressure like propane. It has a lower vapor pressure than propane and therefore propane tanks can be used for storage and transportation. Other characteristics include its energy density, which is lower than conventional diesel fuel it takes 1.8 times as mult volume as diesel to achieve equivalent power output. However, it has a higher cetane number than diesel and hence offers better and more consistent ignition in compression ignition (CD) engines. For the above reasons it is being considered as a fuel for compression ignition direct injection (CIDI) engines.

The following mechanism for DME were obtained

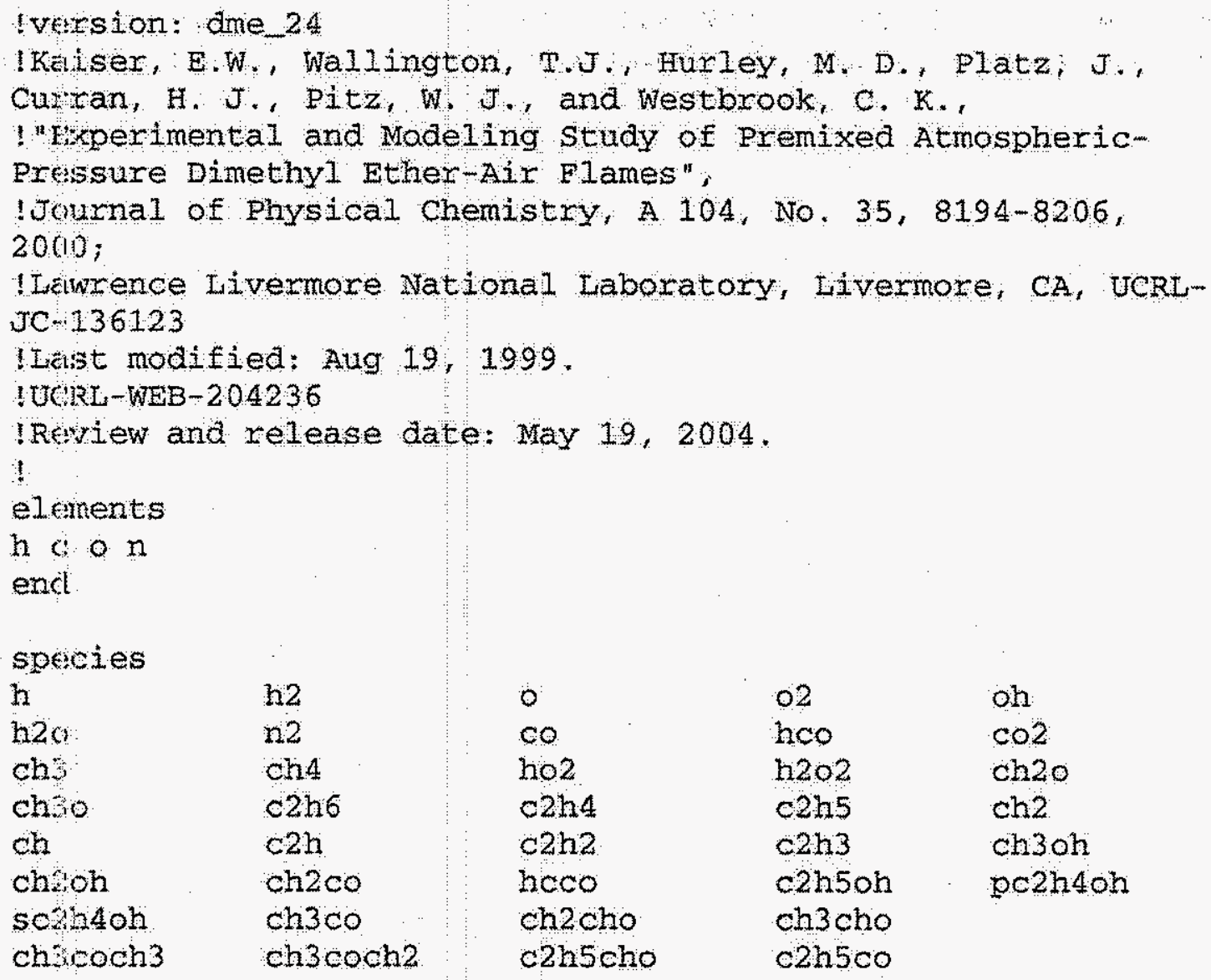

0 o2 on

co heo co2

ho2 h202 ch2o

c2h4 c2h5 ch2

c2h2 c2h3 ch3oh

heco c2h5oh pe2h4oh

ch2cho ch3cho 


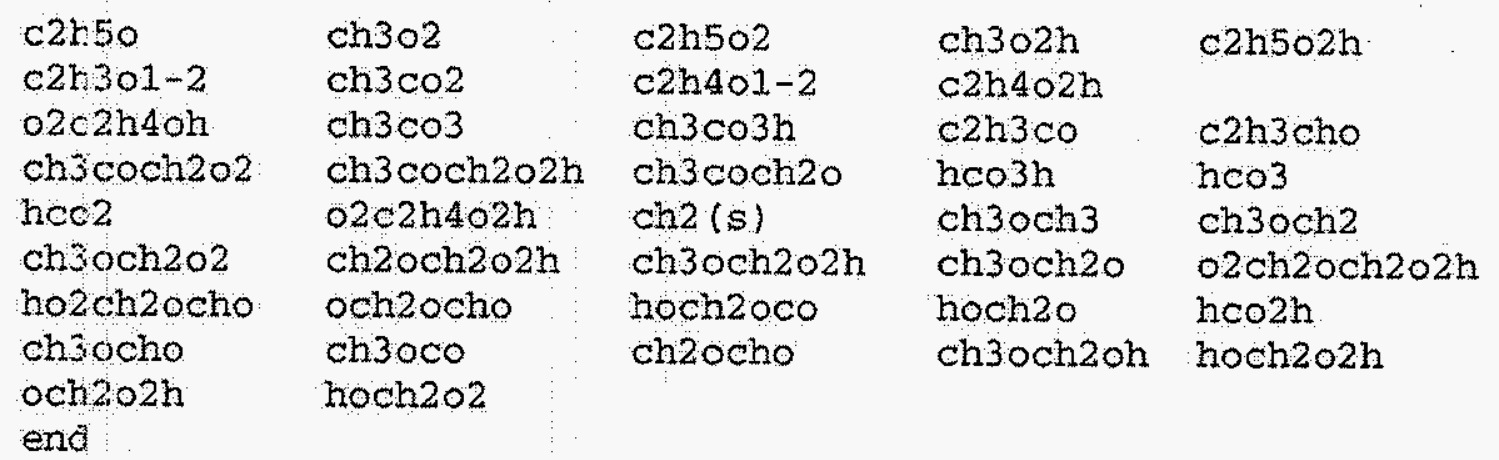

The mechanism is in CHEMKIN format and contains 79 species and 351 reactions. These reactions are the starting point to apply NPCA-NN. Only zero dimensional models will be used to study the evolution of the species. The challenge will be the application of NPCANN to this mechanism, some clever programming will be required to automate the process. Note that to apply NPCA-NN to this mechanism, the reduction modeling will be applied twice. The first application of NPCA-NN will be to develop new reduced variables for the species concentrations. The second application will develop reduced variables for the reacion rates for each species. This way it will not be necessary to use CHEMKIN with KIVA. Zero dimensional CHEMKIN solutions will only be used to generate data for NPCANN training.

\subsection{CFD Code Development and Implementation}

The main effort was to develop and implement a CFD code and to interface the code to CHIMKIN. Initially it was proposed to use the code KIVA. As the project progressed an in house code was developed and used. The process provided valuable experience to the graduate students. The main features of the code were to use kernel function approximation, derivatives approximation using complex variables, and solving the ODEs using the modified Ranga Kutta Method (RKM).

Initially a simple 2-D CFD code was chosen for implementation. The code chosen was the 2-D Euler solver described in the book by Michael Lobo, titled; Time-Marching a stepby-step guide to a flow solver, Ashgate Publishers, 1997. The book contained a detailed description of a two dimensional flow solver with the necessary background mathematics explained in details. The complete code is in FORTRAN programming language. The code was tested on a simple problem, which was to solve for steady flow of air through a plane symnetrical diffuser. In this problem the viscosity and density of air were taken to be $1.91 \mathrm{X}$ $10^{-4} \mathrm{~kg} / \mathrm{m} \cdot \mathrm{s}$ and $1.21 \mathrm{~kg} / \mathrm{m}^{3}$, respectively. The flow profile at the inlet was assumed to be flat with a bulk velocity of $160 \mathrm{~m} / \mathrm{s}$. The flow at the outlet was assumed to be fully developed. The results of the computation visualized in Tecplot are attached. The results are for the grid profle, the Mach number, and static pressure distribution as shown in Figures 6,7 and 8 respectively. 


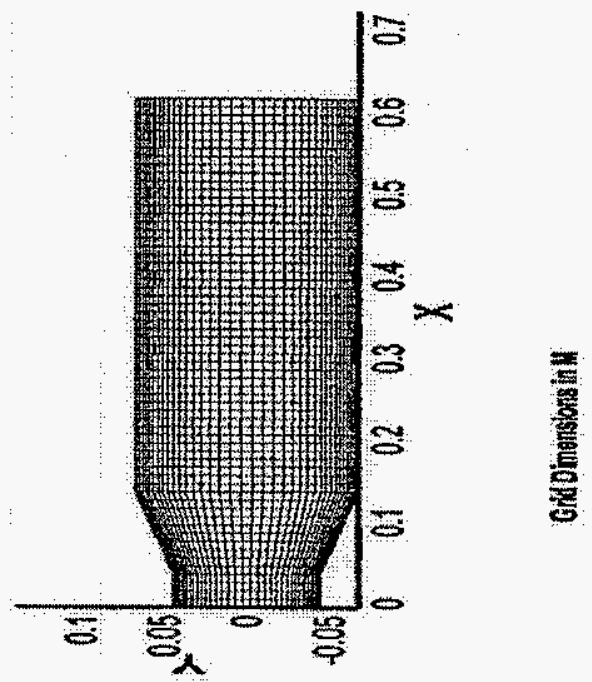

Figure 6. Diffuser Grid Profile

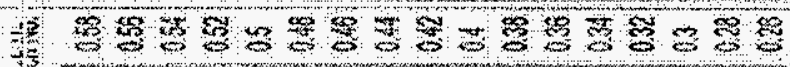

It

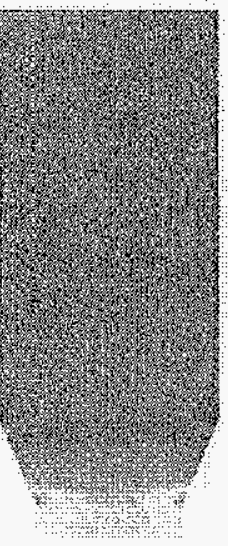

홀

Figure 7. Mach No. Distribution 

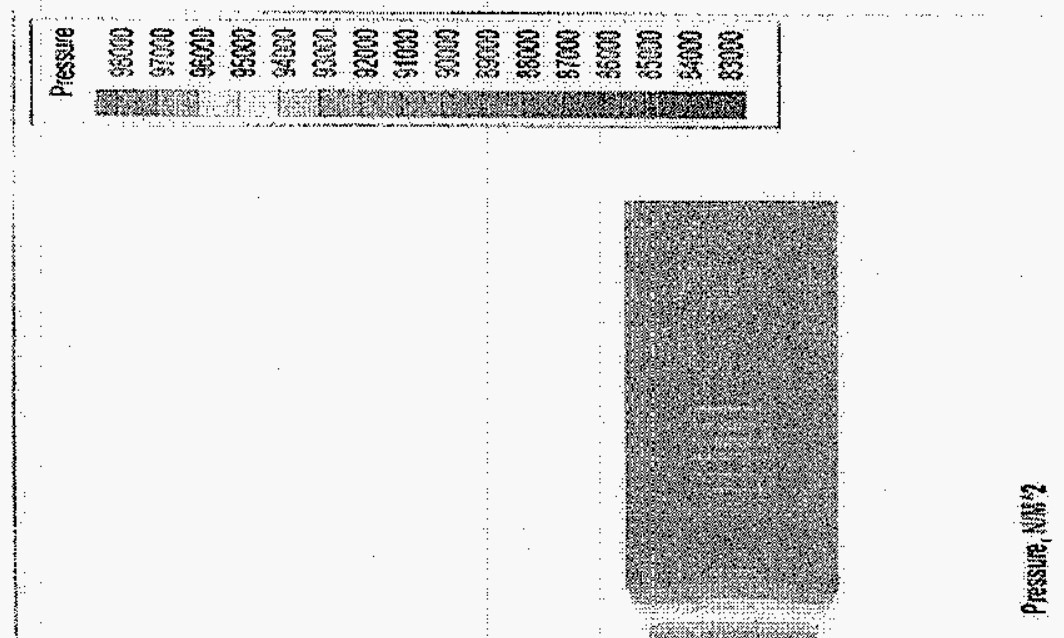

Figure 8. Diffuser Pressure Distribution

The next step in the code development was to extend the code to include reactions. A brief description of procedure of extending the code to include reactions is described below.

The two dimensional Euler equations are expressed in vector form as:

$$
U_{1}+F_{x}+G_{y}=0
$$

where;

$$
U=\left[\begin{array}{c}
p \\
\rho u \\
\rho v \\
\rho E
\end{array}\right] F=\left[\begin{array}{c}
\rho u \\
\rho u^{2}+p \\
\rho u v \\
\rho u h
\end{array}\right] G=\left[\begin{array}{c}
\rho v \\
\rho u v \\
\rho v^{2}+p \\
\rho v h
\end{array}\right]
$$

The primitive yariables are:

$$
\begin{aligned}
& p: \text { density } \\
& u: \text { velocity in } x \\
& y: \text { velocity in } y \\
& p: \text { pressure } \\
& E \text { :energy } \\
& h: \text { enthalpy }
\end{aligned}
$$


There are four partial differential equations (pde's) to solve for six scalar unknown prinitive variables. Two of the unknowns are eliminated by appropriate thermodynamic relationships usually expressed by simple algebraic equations. In order to extend the solver to incorporate additional pde's for simulating reactive flows, the additional equations must be of the same general form as the above. The additional equations will describe the evolution of species concentration. The species equations are written in general cartesian-tensor notation as:

$$
\frac{\partial\left(\rho Y_{i}\right)}{\partial t}+\frac{\partial\left(\rho u_{j} Y_{i}\right)}{\partial x_{j}}+\frac{\partial}{\partial x_{j}}\left(D \frac{\partial Y_{i}}{\partial x_{j}}\right)=\dot{\omega}_{i}
$$

Note that in cartesian-tensor notation, when a subscript is repeated in a term, a summation of two terms (for 2-D) is implied. Therefore $\mathrm{j}=1,2$ and $\mathrm{i}=1$.N, where $\mathrm{N}$ is the total number species. $Y_{i}$ is the mass fraction of specie $i$, and $D$ is the binary diffusion coefficient. This specie continuity equation is derived assuming binary diffusion for all species and that the effective Lewis number is unity. The effective Schmidt number is also assuned to be one. The RHS term $\hat{\omega}$, is the chemical production rate for species $i$. This will be computed directly from CHEMKIN. One more simplification applicable to reactive Euler equition is the assumption that the diffusive coefficient is zero. Replacing $Y_{i}$, with $\left(\rho_{i} / \rho\right)$ abote, the simplified specie continuity equations become:

$$
\frac{\partial\left(\rho_{i}\right)}{\partial t}+\frac{\partial\left(\rho_{i} u_{j}\right)}{\partial x_{j}}=W_{i} \omega_{i} \quad i=1 \ldots N
$$

for $\mathrm{N}$ species, where $W_{i}$ is the nolecular weight of specie $i$. If we assume thermal equilibrium and apply for each partial pressure the ideal gas law

$$
P_{i}=\rho_{i} \frac{R}{W_{i}} T
$$

where $R$ is the universal gas constant and $T$, the static temperature. Using Dalton's law, the total static pressure is:

with

$$
p=\sum_{i}^{N} p_{i}=\rho \frac{R}{W} T
$$

$$
\rho=\sum_{i}^{N} \rho_{i}, Y_{i}=\frac{\rho_{i}}{\rho}, W=\left(\sum_{1}^{N} \frac{Y_{i}}{W_{l}}\right)^{-1}
$$

W will be the molecular weight of the gas mixture. All species will be assumed to be thernally perfect and the specific heats at constant pressure are approximated by polynomials of the form

$$
C_{p_{1}}=A_{i}+B T+C_{i} T^{2}+D_{i} T^{3}
$$


$\mathrm{A}, 3, \mathrm{C}$ and $\mathrm{D}$ are curve fit parameters unique to each specie, $\mathrm{i}$. The static enthalpy, $\mathrm{h}$, is now:

with

$$
h=\sum_{i}^{N} Y_{i} h_{i}(T)
$$

$$
h_{i}(T)=h_{i}^{f}+\int_{T_{s}}^{T_{p}} C_{p_{i}}(T) d T
$$

and using the tabulated data for $h_{i}^{f}$, the heat of formation. Finally the Euler equations to be solved when reactions are present becomes:

$$
U=\left[\begin{array}{c}
\rho_{1} \\
\rho_{2} \\
\rho_{N} \\
\rho u \\
\rho v \\
\rho E
\end{array}\right] F=\left[\begin{array}{c}
\rho_{1} u \\
\rho_{2} u \\
\rho_{N} u \\
\rho u^{2}+p \\
\rho u v \\
\rho u h
\end{array}\right] G=\left[\begin{array}{c}
\rho_{1} v \\
\rho_{2} v \\
\cdot \\
\rho_{N} v \\
\rho v \\
\rho v^{2}+p \\
\rho v h
\end{array}\right]
$$

The code was extended to incorporate these equations with appropriate species prolluction tates for the RIIS of the equations:

A time matching approach for the solver was then extended as a mesh free code. The following is a brief synopsis of 1 ) review of selected time marching literature [Jichao et. al.], and 2) stability considerations.

A time marching integral equation method for unsteady state problems, Jichao $\mathrm{Su}$, Behrouz Tabanok. Computational Methods Applied Mechanical Engineering 142 (1997), pp 203-214.

Most practical problems in engineering take the form of differential equations with the appropriate boundary and initial conditions. Approximating solutions to these problems rely on numerical methods because of the complexity of the boundary and initial conditions, the ireinular geometry, or the nonlinear nature of the problem.

Two commonly used numerical techniques are the finite difference method (FDM) and the finite element method (FEM). The FDM approximates the derivatives in the differential equitions by truncating a Taylor series expansion in terms of the values at a number of discrete mesh points. The result is a set of algebraic equations to which the boundary and initial conditions are applied to approximate the solution of the differential equations. The FEM approximates the unknown functions over each sub domain, called an element, in terms of polynomial interpolation functions. This method also results in a set of algebraic equations 
to which the boundary and initial conditions are applied when the weak formulation is discretized.

In addition to the previous methods, the boundary element method (BEM), also called the boundary integral equation method, is another numerical technique which has been reciving increasing attention among scientists and engineers. It has become an important altemative to FDM and FEM for the solution of engineering problems.

The theory of the boundary integral equation dates to 1903 with Fredholm, who established the existence of solutions on the basis of his limiting discretization procedure. The emergence of computers in the 1950s stimulated the development of numerical methods including the integral equations method. Per the author, Tosaka and Onishi first introduced an approach of implementing time differencing before deriving an integral equation. A similar technique was developed by Su to obtain time marching integral equations for the solutions of two and three dimensional unsteady transonic flows around wings. The main procedures are as follows: 1) time discretization of the differential equations is made by replacing the time derivatives by finite difference, 2) the time discretized differential equation is transformed into an equivalent integral equations by applying the Green's function method, and 3) the integral equation is discretized in space and solved in each time ster using a numerical integration technique. When the BEM is applied to the wave (hyperbolic) and heat conduction (parabolic) equations the same standard form of the semidiscretized differential equations is obtained once the primitive governing differential equations are discretized in time.

The large discretization step method for time-dependent partial differential equations, Zigo Haras, Shlomo Ta'asan, Computers and Fluids 28 (1999), pp.573-602.

The large discretization step method (LDS) is a new method which utilizes a system of low accuracy schemes to approximate a high accuracy discrete approximation of a timedependent differential. The LDS method has been implemented for linear and nonlinear hyperbolic equations. The high and low accuracy schemes are interpreted at the same discretization of a time-dependent operator on fine and coarse grids, respectively. Therefore, a ststem of correction terms and corresponding equations are derived and solved on the coasse grid to yield the fine grid accuracy. The accuracy of the correction terms deteriorates at a tate determined by that grid's discretization. However, since the norm of these terms is significantly smaller than the solution norm, they can be effectively used for many coarse gric time steps. The correction terms are initialized on the fine grid. Then the fine grid has to be a visited to compute new initial data for the correction terms. Initialization of these terms is computationally costly and the LDS approximation consists of more equations that the original problem. On the hand, the large number of time steps performed on the coarse grid before revisiting (the fine grid) renders the algorithm very efficient. The method is very general, simple to implement, and can be used to accelerate existing time marching tecliniques.

Some assumptions include: 1) commutativity, and 2) the selection of an appropriate grid which resolves all the physical frequencies occurring in the problem as well as suitability to long simulation time is assumed.

LDS methods may be viewed as a generalization of the frozen $\tau$ method aimed at accelerating the solution of hyperbolic and parabolic equations, for both implicit and explicit 
timi marching schemes. Hyperbolic equations are investigated since they have not previously been successfully solved using multignid methods. Noted improvements in the LDS method include: 1) significant improvement of the high degree LDS which resulted in a more effisent algorithm that requires low-order intergrid transfers, 2) LDS method was extended to treat nonlinear problems with the same efficiency, and 3) problems with non-periodic boundary conditions solved.

Further investigation is required of the assignment of appropriate boundary conditions to fae corrections terms when the commutativity assumption does not hold. Also, a large errer at the boundary discretization introduced by the one-sided high order interpolation may require selection of differential equations to design appropriate near boundary interpolations with reduced error.

Stability considerations: Very short integration time resulted in an oscillatory error. Increasing by a factor of 10 the $\mathrm{CFL}$ as well as the integration time, eliminates these oscillations, resulting in excellent approximations to the fine grid solution. Higher order dissipation is essential for the algorithm, as lower order dissipations damps too strongly the smeoth components.

Overall, the LDS method discussed is efficient and stable while providing fine grid acciracy by also utilizing a coarse grid. Further investigation is required of the assignment of boundary conditions to the correction term.

Stability Considerations:

Stability depends on the numerical method and on the particular differential equation. For example, if the numerical method introduces errors into calculations at an exponential growth rate as computations proceed then it is unstable. For implicit methods, stability can be maintained over much larger values of delta $t(\Delta t)$ than a corresponding explicit method. Sone implicit methods are unconditionally stable. However, explicit methods, after delta $x$ $(\Delta x)$ is selected, then $\Delta t$ is not and independent choice and is restricted by a prescribed stakility criterion. A method of simplifying partial differential equations (PDE), dissipation, and error analysis leading to widely used stability criterion (of which there are many) are discussed.

A method of simplifying complex PDEs is by first discretizing the spatial operators $(\partial x \partial x x$, etc) on a chosen grid. This converts the PDE into a system of ordinary differential equations (ODE) to which an appropriate time integration method is applied to obtain a numerical solution. It is important to note that a single ODE system is not being considered but rather a family of systems which are parameterized by the grid parameter, step-size.

A stability consideration is the introduction of dissipation. Dissipation is introduced becuse of two reasons. First, it can eliminate the high frequency terms. Second, when solvtions with discontinuities arise, dissipation can suppress undesirable oscillations. If a hyperbolic PDEs is consistent and has dissipative terms of any order $\rho>0$, then the app oximation is stable. Parabolic PDEs are dissipative by nature. Their approximation is alse dissipative. Therefore, if the approximation of the parabolic equation is parabolic, then the approximation is stable.

Sources of error analysis are a means to analyze the stability characteristics of a given PDI Errors are propagated from one time-step to the next. One error type is the discretization error which is the difference between the exact analytical solution of the PDE and the exact (without any rounding) solution of the corresponding difference equation. In 
other words, this is the truncation error for the PDE plus any errors introduced by the numerical treatment of the boundary conditions. A second error type is the round-off error whith is a numerical error introduced after a repetitive number of calculations in which there is rounding to some significant figure.

John von Neumann's analysis of the above errors associated an amplification factor (AF) with the algorithm. It provides an indication of how errors will behave in the course of the solution procedure, If the $|A F|<1$, where $|A F|=\left|\frac{E r r o r}{E r r o r}\right|$, errors will eventually disappear. Otherwise, the errors may grow without bound and the algorithm will become unstable.

Other analysis of errors was conducted by Courant, Friedrichs, and Lewy (CFL). For hyp rbolic equations, CFL showed that a numerical scheme could not be consistent if the numerical domain of dependence did not contain the physical domain of dependence. The notim of consistency of a numerical scheme refers to the ability to approach the exact solution to a differential equation as the mesh size tends to zero. The CFL condition does not guarantee stability, except in cases with simple schemes. However, any scheme not satisfying the CFL condition will be unstable. The algorithm becomes unstable if the $\mathrm{CN}$ exceeds a certain critical value which may be difficult to evaluate even for a simple equation. Therefore, assuming a spatial grid is fixed, $\Delta \mathrm{t}$ must be chosen such that $\mathrm{CN}=\sigma=\lambda \Delta \mathrm{t} / \Delta \mathrm{x}<\mathrm{x}$ or $\Delta t=\Delta x / \lambda$. If $\lambda>0$ is assumed, a more refined grid restricts time-step greater. If $\lambda<0$ is assumed, then the CN will be negative and the scheme will always be unstable.

In reaching the overall goal of simulating a two-dimensional solver implementing novel and accurate methods, functions were approximated using a kemel, the derivatives were approximated using complex variables methods, and then the ordinary differential equations (ODE) were solved with time-marching approach with a modified $4^{\text {th }}$ order Runge-Kutta Method (RKM). The three aforementioned methods have been implemented separately and are now being combined into one code to solve the Euler equations of fluid flow.

\subsection{Meshfree Techniques}

The problem of time marching function approximated solutions inherent in emerging meshifree Computational Fluid Dynamies (CFD) solution techniques is then addressed. These numerical solutions of partial differential equations (PDE) of CFD have been dominated by either finite difference methods, finite element methods, or finite volume methods. These methods can be derived from the assumptions of the Taylor expansion based loca interpolation schemes and they require a mesh to support the local approximation. The problem is that in complex shaped domains, the construction of the mesh is a non-trivial problem. Typically with these methods, only the function is continuous across meshes, but not ts partial derivatives. The difficulties of mesh construction and discontinuous derivatives have led to the development of mesh independent methods or meshfree (MF) methods. These new MF methods represent the next generation of CFD solvers as they mature In these metlods the local function approximation method is independent of the mesh (or design points) of the geometric domain in which a solution is sought. The approximation of the loca function by the kernel based statistical methods is sought. It is shown, that the approximated solution in an arbitrary mesh can be matched in time to obtain the steady state and/or time dependent solution of the PDE. To differentiate the function, the novel approach of complex variables method (CVM) is used. Once the derivatives have been obtained, then 
an aspropriate time marching approach can be chosen and implemented to solve the resulting system of ODEs. In the current work the standard modified fourth order Runga-Kutta (RK) approach was used to march the solution in time.

\subsection{Grids}

Aspect ratios, uniformity, and orthogonality were non-issues in spite of the complexity of the problem being solved. However, calculating realistic dimensions for the geometry of the somputational domain proved to be a challenging task. Since the fluid flow is turbulent, the Reynolds number, which is the ratio of inertial forces to viscous forces, was not the appropriate tool to calculate the geometry. Instead, mass flow rate was utilized. Mass flow rate is defined as the movement of mass per time. The formula is given as,

$$
m=p V A
$$

whe

$$
\begin{aligned}
& m=\text { mass flow rate } \\
& \rho=\text { density } \\
& V=\text { velocity } \\
& A=\text { flow cross-sectional area },
\end{aligned}
$$

An analysis of the geometry utilized in Lobo (1997) aided in the assumption to let the $x$ direstion length equal to twice the $y$-direction length. If the $y$-direction length $=\mathrm{L}$ and the $\mathrm{x}$ dire tion length $=2 \mathrm{~L}$, then the cross-sectional area is $A=2 \mathrm{~L}^{2}$. Then, fluid (air) velocity is equal to $678.1 \mathrm{~m} / \mathrm{s}$ and density is equal to $1.23 \mathrm{~kg} / \mathrm{m}^{3}$. In addition, the mass flow rate is set equal to 10 based on isentropic supetsonic flow examples found in gas dynamics textbooks and literature. After substituting these values into the mass flow formula, equation 5,1 , the computed $\mathrm{x}$-direction and $\mathrm{y}$-direction lengths are approximately 0.0490 meters and 0.0245 metors respectively. However, when analyzing the final results it is necessary to use a different grid to facilitate the comparison with a proven solver. Figure 9 is an illustration of the grid used in the final comparison. The mesh is a rectangular sheet with a bump in the middle. Even though it is a structured mesh, the computations are not affected because kerrel approximation methods (KAM) can be used with either mesh or mesh free. In addilion, this geometry is more desirable because the flow dynamics will be more evident.

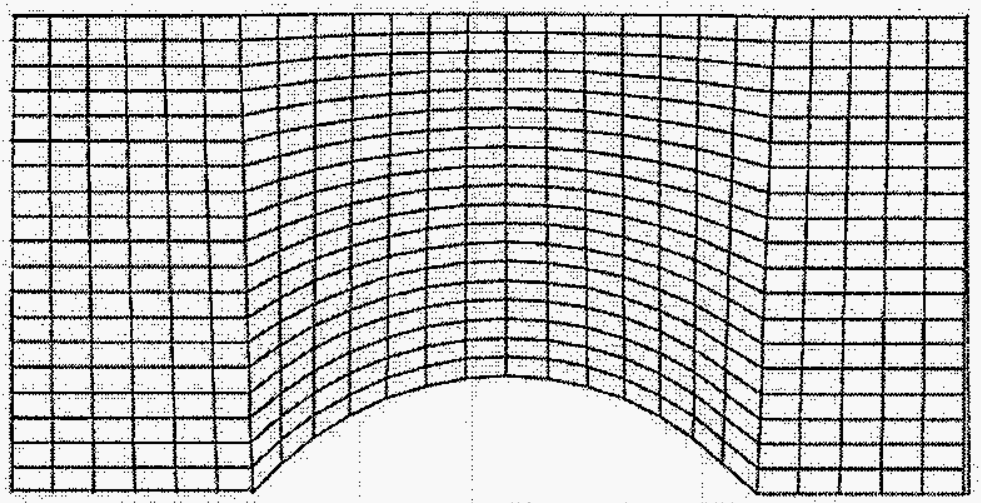

Figure 5.1.1 Mesh: Two Dimensional Flow Over A Cascade

Figure 9. Grid Distribution Used in Final Comparison 
In addition, after the code components had been incorporated into the preliminary version but before the mesh (Figure 9 ) was substituted, a minor adjustment was made. Initially the first MF grid (using uniform lengths) and the second MF grid (using midpoints) contained a different number of nodes (ordered pairs). Therefore, approximating the functional values usirg a disproportionate number of points would result in an inaccurate and unreliable conputation. Consequently, the number of nodes of both of these grids was set equal to each other so as to not skew the results. In addition, the code was modified to alternate the KAM and CVM computations between the mesh one and mesh two. These two adjustments were mace to ensure that the most recently calculated values are utilized as input for the next timester.

\subsection{Boundary Conditions}

Boundary conditions (BC) are discussed in general and then specifically as they pertain to the current project. Even though the flow is modeled by the same governing equations, the flow behaves differently depending on whether it is traveling at subsonic or supersonic spewd. Here, the specification of the BCs is critical. The BCs are summarized as follows.

In general, $\mathrm{BCs}$ are given by assigning a value to the total pressure at inlet, the temperature at inlet, and a third condition either at the inlet or outlet depending on whether the Mach number (subsonic or supersonic). A simpler altemative is an initial guess which assigns constant values throughout the flow field to density $(\rho)$, velocity ( $u$ and $v$ ), and pressure $(p)$. In addition, if the flow is supersonic the condition specified is the Mach number However, if the flow is subsonic the condition specified is the static pressure at the out et. Values for the variables are deternined by the following formulae.

$$
\begin{aligned}
p & =p_{0} f(\gamma, M) \\
T & =T_{0} f(\gamma, M) \\
p & =\frac{p}{R T} \\
c & =\sqrt{\gamma R T} \\
q & =M c \\
\text { Whore, } & \\
p & =\text { static pressure } \\
p_{0} & =\text { stagnation pressure } \\
\gamma & =\text { ratio of specific heats } \\
M & =\text { Mach number } \\
T & =\text { static temperature } \\
T & =\text { stagnation temperature } \\
\rho & =\text { density } \\
R & =\text { gas constant } \\
c & =\text { speed of sound } \\
u & =x \text {-direction velocity } \\
v & =\text { y-direction velocity } \\
q & =\sqrt{u^{2}+v^{2}}=\text { total velocity }
\end{aligned}
$$


If we assume that $T_{0}$ and $P_{0}$ at the inlet remain constant, then the Mach number and velocities are calculated as below.

$$
M=\sqrt{\frac{2}{\gamma-1}\left[{\frac{p_{0}}{p}}^{\frac{\gamma-1}{\gamma}}-1\right]}
$$

At a supersonic inlet, four physical BCs must be specified; they are $T_{0}$ (stagnation temperature), $p_{0}$ (stagnation pressure), a physical condition $\rho$ (density), and $M$ (Mach number). However, at the supersonic outlet, all of the BCs are imposed numerically. Typically, $\rho$ (density), $u$ (x-direction velocity), $v$ ( $y$-direction velocity, and $p$ (pressure) are extrupolated linearly from interior points.

Then, temperature is calculated by solving for $T$ using the equation of state $p=\rho R T$. This yield

$$
T=\frac{p}{\rho R}
$$

Energy, $e$ and, enthalpy, $h$ are calculated using the following equations

$$
\begin{aligned}
& e=c_{\nu} T+\frac{1}{2}\left(u^{2}+v^{2}\right) \\
& h=c_{p} T+\frac{1}{2}\left(u^{2}+v^{2}\right)
\end{aligned}
$$

Where $c_{y}$ and $c_{p}$ are the specific heats at constant volume and constant pressure.

$A t$ the subsonic inlet, $T_{0}$ (stagnation temperature), $p_{0}$ (stagnation pressure), and a third physical condition are specified. However, at the subsonic outlet it is usual to specify the statio pressure, $p$. p (density), $u$ ( $\mathrm{x}$-direction velocity), $v$ (y-direction velocity) are linearly extrapolated from interior points.

Ii practice, the BCs at the supersonic inlet are $T$ (temperature), $q$ (total velocity), $c$ (steed of sound). Temperature is calculated based on the following formula.

$$
T=T_{0}\left[\frac{1}{1+\frac{\gamma-1}{2} M^{2}}\right]
$$

The speed of sound and total velocity are calculated as below.

$$
\begin{aligned}
& c=\sqrt{\gamma R T} \\
& q=M c
\end{aligned}
$$

Hovever, in practice at the subsonic inlet $u$ ( $x$-direction velocity), $v$ (y-direction velocity), and $q$ (total velocity) are extrapolated $c$ (speed of sound) is calculated as below.

$$
c=\sqrt{\gamma R T}
$$

$T$ (emperature) is calculated using the following

$$
T=T_{0}-\frac{1 q^{2}}{2}
$$

and $M$ (Mach number) is calculated as follows. 


$$
M=\frac{q}{c}
$$

In practice, the upper and lower wall $\mathrm{BCs}$ are assigned in the same manner. Here there is only one physical $\mathrm{BC}$ governing the velocity components. This is the tangency condition which states that the velocity vector at any point on the wall must be tangent to the surface. Therefore, $u(x$-direction velocity) and $v$ ( $y$-direction velocity) may be assigned any values and still satisfy the tangency condition. In other word, since there is no velocity component perpindicular to the wall, the velocity values at the wall will not affect the velocity values in the interior points. And therefore will not affect the mainstream solution. However, pressure is affected. Hence, $y$ ( $y$-direction velocity) is assigned a zero value. Then $u$ (x-direction velodity) is calculated by extrapolating $q$ (total velocity) from the interior and separating $q$ into ts components as follows.

Using the definition of total velocity as stated below.

$$
q=\sqrt{u^{2}+v^{2}}
$$

Substituting $v=0$ yields $q=\sqrt{u^{2}+(0)^{2}}$ which simplifies to $q=u$.

The pressure $B C$ is handled differently since it does affect the mainstream solution. Analysis of the pressure tangency condition for an inviscid flow follows. Here, the pressure grad ent nomal to the wall surface at any point can be expressed in terms of the tangential velosity and the radius of curvature. Radius of curvature, $R_{c}$ is defined as the rate of change of the direction with respect to the arch length.

$$
R_{c}=\frac{d \theta}{d s}
$$

On the walls, the total velocity and the tangential velocity are the same because the radius of curvature for a flat wall is zero. Therefore, the pressure gradient, $\frac{d p}{d y}$, normal to the wall is also zero. This means that the pressure at the wall can be assigned the value of the interior point closest to it (in an inward perpendicular direction).

For a curved wall, the pressure gradient can be used to extrapolate to the wall using the follc wing formula.

$$
p_{n}=\frac{\rho q^{2}}{R_{c}}
$$

\subsection{Kermel Methods}

Non-parametric regression, also known as local regression modeling, consists of fitting. a curve to a data set where there is little or no knowledge about its shape The basic idea behind local regression consists of obtaining the prediction for a data point $\mathrm{x}$ by fitting a function locally. Kernel methods can be used to approximate functions on either structured or unstructured grids. Given is a data set of the form $\{(\mathrm{Xi}, \mathrm{Yi}), i=1,2, \ldots \mathrm{n}, \mathrm{i} \in \mathrm{N}\}$ which is assumed to occur in predictor-response pairs. The Gaussian kemel used to approximate a function $f(x, y)$ where $x \in[a ; b]$ and $y \in[c, d]$ is 


$$
f_{\text {estimate }}(x, y)=\frac{\sum_{i=1}^{n} h_{i} Y_{i}}{\sum_{i=1}^{n} h_{i}}
$$

where

$$
\begin{aligned}
& h_{f}=e^{\frac{D_{i(x)}^{2}+D_{i(y)}^{2}}{2 \sigma^{2}}} \\
& D_{i(x)}^{2}=\left(x-X_{i}\right)^{T}\left(x-X_{i}\right), \\
& D_{i(y)}^{2}=\left(y-Y_{i}\right)^{T}\left(y-Y_{i}\right)
\end{aligned}
$$

and

$$
\sigma=0.100 .
$$

The use of kemels to approximate the EULER functions made the grid generation a relatively easy task. Aspect ratios, uniformity, and orthogonality were non-issues in spite of the somplexity of the problem being solved. However, coming up with a realistic geometry for the computational domain proved to be a challenging task

\subsection{Complex Variables Method for Obtaining Derivatives}

Complex variables approach was easily implemented to obtain derivatives. Given a complex number $z=x+1$, we can write the complex function $f(z)$ as

$$
f(z)=u(x, y)+i v(x, y)
$$

where $u$ and $v$ are real valued functions. Using the basic definition of derivative, we can write

$$
f^{\prime}(z)=\lim _{h \rightarrow 0} \frac{f(z+h)-f(z)}{h}
$$

$h$ can be both real and imaginary. Now as $h \rightarrow 0$ through real values, the limit $f^{\prime}(z)$ must be the same for both approaches of $h$ to zero. First if $h$ is teal,

Now if $h$ is imaginary

$$
\begin{aligned}
\lim _{h \rightarrow 0} \frac{f(z+h)-f(z)}{h} & =\lim _{h \rightarrow 0} \frac{u(x+h, y)-u(x, y)}{h}+i \lim _{h \rightarrow 0} \frac{v(x+h, y)-v(x, y)}{h} \\
& =\frac{\partial u}{\partial x}+i \frac{\partial v}{\partial x}
\end{aligned}
$$

$$
\begin{aligned}
\lim _{h \rightarrow 0} \frac{f(z+i h)-f(z)}{h} & =\lim _{h \rightarrow 0} \frac{u(x, y+h)-u(x, y)}{i h}+i \lim _{h \rightarrow 0} \frac{v(x, y+h)-v(x, y)}{i h} \\
& =\frac{\partial v}{\partial y}-i \frac{\partial u}{\partial y}
\end{aligned}
$$

Since the limit $f^{\prime}(z)$ must be the same, we obtain

$$
\frac{\partial u}{\partial x}=\frac{\partial v}{\partial y} \text { and } \frac{\partial u}{\partial y}=-\frac{\partial v}{\partial x}
$$

whith are the Cauchy-Riemann equations. From the first equation 


$$
\frac{\partial u}{\partial x}=\lim _{h \rightarrow 0} \frac{v(x, y+h)-v(x, y)}{h}
$$

and on the real axis $y=0, u(x)=f(x)$ and $v(x)=0$, therefore for real functions

$$
\frac{\partial u}{\partial x}=\lim _{h \rightarrow 0} \frac{v(x, h)-v(x)}{h}
$$

which can now be written as

$$
\frac{\partial f}{\partial x}=\lim _{h \rightarrow 0} \frac{\operatorname{Im}[f(x+i h)]}{h}
$$

This equation is then the basis for the Complex Variables Method (CVM) of derivatives. Any code that computes any given function can be made to compute the first derivatives of the function by simply converting the code to tun using complex variables. It can be shown via a Taylor series expansion that the estimated derivatives are of $O\left(h^{2}\right)$.

Initially the design grid and the mesh free (randomly generated) grid contained a different number of nodes (ordered pairs). The mesh free grid contained fewer nodes than the design grid. Therefore, approximating the functional values on the design gid using far less nodss as input in this approximation would result in inaccurate and unreliable computations. Consequently, the code was modified to alternate the Complex Derivative computation between the design grid and the randomly generated (mesh free) grid. Also, the number of nodes of both of these grids was set equal to each other so as to not skew the results. These minor adjustments ensure that the most recently calculated values are utilized as input for the next time-step.

\subsection{Runge-Kutta Method(RKM)}

Consider an ODE of the form:

$$
\frac{d y}{d x}=f(x, y)
$$

The RKM computes the value of $f(x, y)$ at strategic points in the rectangle bounded by the points $[x, x+h, y(x), y(x+h)]$ and then combines them in such a way so as to increase the order of accuracy. The formula involves a weighted average of values of $f(x, y)$ inside this domain. In order to determine a point in this rectangle, it is necessary to compute the unknown $y(x+\alpha h)$ where $\alpha$ is a coefficient between 0 and 1 . The RK scheme involves several stages or applications As each stage is added, the order of accuracy increases by 1 . Selection of the coefficient is cumbersome. These may be obtained by straightforward Taylor series expinsion. More information can be obtained from numerical analysis textbooks.

The most widely used and most successful RKM is the fourth order scheme:

$$
\begin{aligned}
& f_{1}=f(x, y) \\
& f_{2}=f(x+1 / 2 h, y+1 / 2 h f) \\
& f_{3}=f\left(x+1 / 2 h, y+1 / 2 h f_{2}\right) \\
& f_{4}=f\left(x+h, y+h f_{3}\right) \\
& y(x+h)=y(x)+h\left[f_{1}+2 f_{2}+2 f_{3}+f_{4}\right] / 6
\end{aligned}
$$

An m-stage modified RK scheme which is slightly different from standard formulation is implemented because it requires less array storage: 


$$
\begin{aligned}
& y_{1}=y(x)+\alpha_{1} h f(y) \\
& y_{2}=y(x)+\alpha_{2} h f\left(y_{1}\right) \\
& \cdots \\
& y_{m-1}=y(x)+\alpha_{m-1} h f\left(y_{m-2}\right) \\
& y(x+h)=y_{m}=y(x)+\alpha_{m} h f\left(y_{m-1}\right)
\end{aligned}
$$

Both of the above RK methods use the classical approach and add steps between $y(x)$ and $y(x+h)$. They achieve increased accuracy due to weighting more recent calculations. In general, the RKM is preferred to a Taylor series formula of same order accuracy because the RKMI does not require that the higher partial derivatives be computed. Furthermore, the RKU is a very accurate formula, since halving the time-step reduces the local formula error by the factor 1/32. (A Fortran code was written to implement the modified RKM)

\subsection{The Euler Equations of flow in two-dimensional form. Steady, Isentropic:}

The following shows how to obtain the pure form of the flux components using a solution vector and thermodynamic relationships for steady isentropic form. The unsteady formi is described below.

The Euler equations in vector form for a two-dimensional flow are

$$
U=\left(\begin{array}{l}
\rho \\
\rho u \\
\rho v \\
\rho e
\end{array}\right), \quad F=\left(\begin{array}{l}
\rho u \\
\rho u^{2}+p \\
\rho u v \\
\rho u h
\end{array}\right) \quad \text { and, } \quad G=\left(\begin{array}{l}
\rho v \\
\rho u v \\
\rho v^{2}+p \\
\rho v h
\end{array}\right)
$$

Written in generic form the same are $\frac{\partial U}{\partial t}+\frac{\partial F}{\partial x}+\frac{\partial G}{\partial y}=J$.

When the flow is steady and isentropic, the generic form reduces to:

$$
\frac{\partial F}{\partial x}+\frac{\partial G}{\partial y}=0 \text { Because the source term } \mathrm{J}=0 \text { and it is assumed that } \frac{\partial U}{\partial t}=0
$$

Then the $F$ vector is treated as the solution vector. In order to minimize the oscillations which can occur when obtaining the solution to the Euler equations of flow using a numerical method, the primitive variables must be decoded using the fluxes $F_{1}, F_{2}, F_{3}$, and $F_{4}$. The $G$ flux components are also restated using the $\mathrm{F}$-flux components which are:

$$
\begin{aligned}
& F_{1}=\rho u \\
& F_{2}=\rho u^{2}+p
\end{aligned}
$$




$$
\begin{aligned}
& F_{3}=\rho u v \\
& F_{4}=p u\left[e+\frac{u^{2}+v^{2}}{2}\right]+p u
\end{aligned}
$$

The following solves for the primitive variables in terms of the fluxes $F_{1}, F_{2}, F_{3}$, and $F_{4}$. Solving for u yields

Solving for $p$ yields

$$
u=\frac{F_{1}}{\rho}
$$

$$
p=F_{2}-\frac{F_{1}^{2}}{\rho}
$$

Solving for $\mathrm{v}$ yields.

$$
\nu=\frac{F_{3}}{F_{1}}
$$

In aldition, the following thernodynamic relations will be utilized in this derivation.

Substatuting

$$
e=\left(\frac{1}{y-1}\right) \frac{p}{\rho}
$$

$$
p=F_{2}-\frac{F_{1}^{2}}{\rho}
$$

simplifying e can be written as,

$$
e=\left(\frac{1}{\gamma-1}\right)\left(\frac{F_{2}}{\rho}-\frac{F_{1}^{2}}{\rho^{2}}\right)
$$

Substituting values and rearranging we get;

$$
F_{4}=\rho\left(\frac { F _ { 1 } } { \rho } \left[\left(\frac{1}{\gamma-1}\right)\left(\frac{F_{2}}{\rho}-\frac{F_{1}^{2}}{\rho^{2}}\right)+\frac{1}{2}\left(\left(\frac{F_{1}}{\rho}\right)^{2}+\left(\frac{F_{3}}{F_{1}}\right)^{2}\right]+\left(F_{2}-\frac{F_{1}^{2}}{\rho}\right)\left(\frac{F_{1}}{\rho}\right)\right.\right.
$$

Further simplifying the above substitutions yields,

$$
F_{4}=\frac{F_{1} F_{2}}{(\gamma-1) \rho}-\frac{F_{1}^{3}}{(\gamma-1) \rho^{2}}+\frac{F_{1}^{3}}{2 \rho^{2}}+\frac{F_{3}^{2}}{2 F_{1}}+\frac{F_{1} F_{2}}{\rho}-\frac{F_{1}^{3}}{\rho^{2}}
$$

Multplying both sides of the equation by $\rho^{2}$, then

$$
F_{4} \rho^{2}=\frac{F F_{2}}{(\gamma-1)} \rho-\frac{F_{1}^{3}}{(\gamma-1)}+\frac{F_{1}^{3}}{2}+\frac{F_{3}^{2}}{2 F_{1}} \rho^{2}+F_{1} F_{2} \rho-F_{1}^{3}
$$

Grouping like terms yields, 


$$
\left(\frac{F_{3}^{2}}{2 F_{1}}-F_{4}\right) \rho^{2}+\left(\frac{F_{1} F_{2}}{(\gamma-1)}+F_{1} F_{2}\right) \rho-\left(\frac{F_{1}^{3}}{(\gamma-1)}+\frac{F_{1}^{3}}{2}\right)=0
$$

Simplifying the coefficient of $\rho$ and the constant term yields the following,

$$
\left(\frac{F_{3}^{2}}{2 F_{1}}-F_{4}\right) \rho^{2}+\left(\frac{\gamma}{(\gamma-1)} F_{1} F_{2}\right) \rho-\frac{(\gamma+1)}{2(\gamma-1)} F_{1}^{3}=0
$$

Density, $\rho$, can now be solved for using the quadratic formula.

$$
\begin{aligned}
& \rho=\frac{-B \pm \sqrt{B^{2}+4 A C}}{2 A} \\
& A=\frac{F_{3}^{2}}{2 F_{1}}-F_{4}, \\
& B=\frac{\gamma}{(\gamma-1)} F_{1} F_{2}, \\
& C=-\frac{(\gamma+1)}{2(\gamma-1)} F_{1}^{3}
\end{aligned}
$$

The G-flux components can now be restated in pure form as follows:

$$
\begin{aligned}
& G_{1}=\rho \frac{F_{3}}{F_{1}} \\
& G_{2}=F_{3} \\
& G_{3}=\rho\left(\frac{F_{3}}{F_{1}}\right)^{2}+F_{2}-\frac{F_{1}^{2}}{\rho} \\
& G_{4}=\frac{\gamma}{\gamma-1}\left(F_{2}-\frac{F_{1}^{2}}{\rho}\right)^{2} \frac{F_{3}}{F_{1}}+\frac{\rho}{2} \frac{F_{3}}{F_{1}}\left[\left(\frac{F_{1}}{\rho}\right)^{2}+\left(\frac{F_{3}}{F_{1}}\right)^{2}\right]
\end{aligned}
$$

5.8 The Euler Equations of flow in two-dimensional form: Unsteady, Isentropic: obtaining the pure form of the flux components in terms of the solution vector $U$.

Restating the Euler equations in vector form for a two-dimensional flow are 


$$
U=\left(\begin{array}{l}
\rho \\
\rho u \\
\rho v \\
\rho e
\end{array}\right), \quad F=\left(\begin{array}{l}
\rho u \\
\rho u^{2}+p \\
\rho u v \\
\rho u h
\end{array}\right) \quad \text { and, } \quad G=\left(\begin{array}{l}
\rho v \\
\rho w \\
\rho v^{2}+p \\
\rho v h
\end{array}\right)
$$

Written in generic form the same are $\frac{\partial U}{\partial t}+\frac{\partial F}{\partial x}+\frac{\partial G}{\partial y}=J$

Since the flow is isentropic the source term $J=0$. The solution vector is $U$ and the $F$ and $G$ components must be expressed algebraically in term of the solution vector components.

$$
\begin{aligned}
& U_{1}=\rho \\
& U_{2}=\rho u \\
& U_{3}=\rho v, \text { and } \\
& U_{4}=\rho e .
\end{aligned}
$$

The fundamental vatiables then are:

Solving for $\rho: \rho=U_{1}$.

Solving for $u=\frac{U_{2}}{U_{1}}$.

Solving for $v: v=\frac{U_{3}}{U_{1}}$

And, solving for $\mathrm{e}: e=\frac{U_{4}}{U_{1}}$.

Using the following thermodynamic relationship for pressure, $p$, and substituting the fundamental variables yields

$$
p=\rho(\gamma-1)\left[e-\frac{1}{2}\left(u^{2}+v^{2}\right)\right]=U_{1}(\gamma-1)\left[\frac{U_{4}}{U_{1}}-\frac{1}{2}\left(\frac{U_{3}^{2}}{U_{1}^{2}}+\frac{U_{3}^{2}}{U_{1}^{2}}\right)\right]
$$

Finally, substituting into the components of the flux $\mathrm{F}$ and $\mathrm{G}$ vectors yields:

$$
F_{1}=U_{2}
$$




$$
\begin{aligned}
& F_{2}=\frac{U_{2}^{2}}{U_{1}}+U_{1}(\gamma-1)\left[\frac{U_{4}}{U_{1}}-\frac{1}{2}\left(\frac{U_{2}}{U_{1}^{2}}+\frac{U_{3}^{2}}{U_{1}^{2}}\right)\right] \\
& F_{3}=\frac{U_{2} U_{3}}{U_{1}} \\
& F_{4}=\frac{U_{2} U_{4}}{U_{1}}+U_{2}(\gamma-1)\left[\frac{U_{4}}{U_{1}}-\frac{1}{2}\left(\frac{U_{2}{ }^{2}}{U_{1}^{2}}+\frac{U_{3}{ }^{2}}{U_{1}^{2}}\right)\right] \\
& G_{1}=U_{3} \\
& G_{2}=\frac{U_{2} U_{3}}{U_{1}}=F_{3} \\
& G_{3}=\frac{U_{3}^{2}}{U_{1}}+U_{1}(\gamma-1)\left[\frac{U_{4}}{U_{1}}-\frac{1}{2}\left[\frac{U_{2}{ }^{2}}{U_{3}^{2}}+\frac{U_{3}{ }^{2}}{U_{1}^{2}}\right)\right] \\
& G_{4}=\frac{U_{3} U_{4}}{U_{1}}+U_{3}(\gamma-1)\left[\frac{U_{4}}{U_{1}}-\frac{1}{2}\left(\frac{U_{2}{ }^{2}}{U_{1}^{2}}+\frac{U_{3}{ }^{2}}{U_{1}^{2}}\right)\right]
\end{aligned}
$$

\subsection{Solving the ODEs Using the RKMI}

A modified RKM was incorporated to solve the ODEs. The RKM and boundary conditions (BC) were the key elements in this, the Time-Marching portion of the code. The BC at the supersonic inlet were specified since the characteristic curves of the EULER equinions were in the computational domain. The BCs at the supersonic outlet were imposed numcrically using linear interpolation. The lower wall BCs were mixed in nature. The velccity in the y-direction, $\mathrm{V}$ was set equal to 0 , density was linearly interpolated, and the other variables were calculated using thernodynamic relationships. The top wall BCs were calculated using linear interpolation.

\subsection{Comparison with Lobo Solver}

The current thesis Euler solver with novel methods is compared against Lobo's [1997] Euler solver which is a traditional solver utilizing the fundamental CFD methods: structured mest, dervative approximation using differencing, and ODE solution using the same molified $4^{\text {th }}$ order Runge-Kutta method utilized in the current thesis.

Higures 5.3 through 533 illustrate results after 30000 time steps using Lobo's (1997) solver Even after 40000 time steps, the code did not converge. Results for the current thesis solver are forthcoming. 

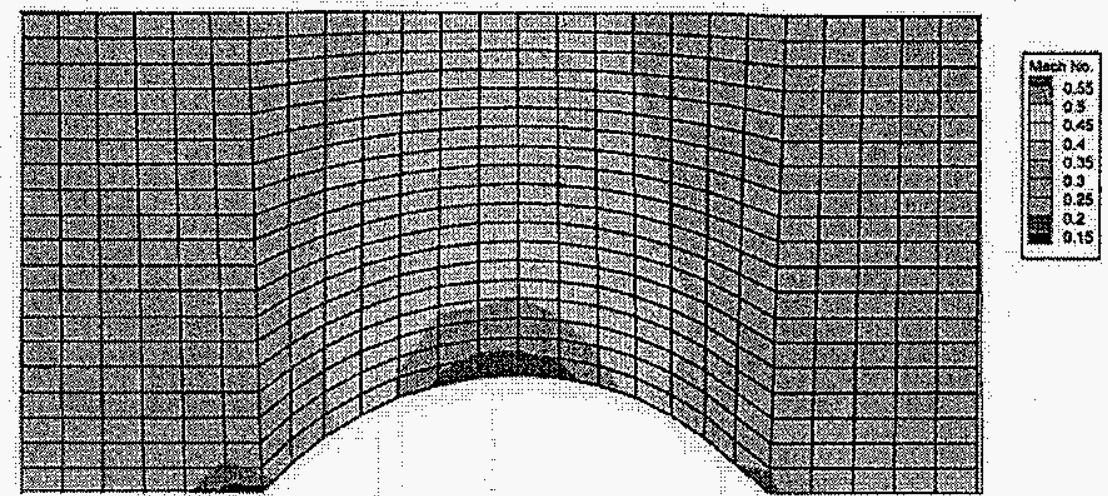

Figure 5.3.1 Lobo Mach Number Results, 30000 Time Steps

Figure 10. Lobo Mach Number Results after 30000 Timé Steps

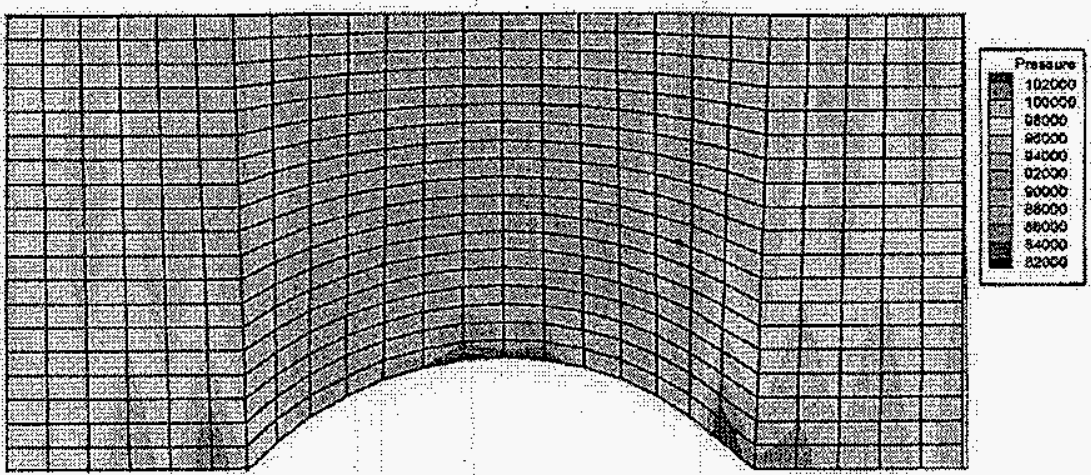

Figure 5.3.2 Lobo Pressure Results, 30000 Time steps

Figure 10. Lobo Pressure Results after 30000 Time Steps

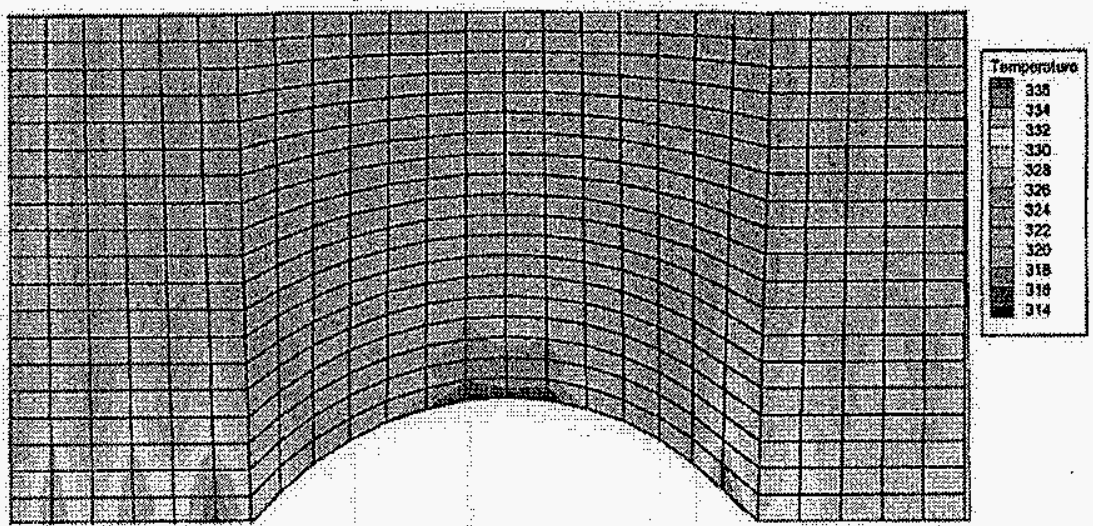

Figure 5.3.3 Lobo Temperature Results, 30000 Time Steps

Figure 10. Lobo Temperature Results after 30000 Time Steps 


\subsection{Conclusions}

The following conclusions could be drawn from the completion of the project:

- Multilayer Perceptron Neural Network (MLP-NN) was modified to implement NPCA-NN.

- The training rate of NPCA-NN was improved with the Generalized Regression Neural Network (GRNN) based on kemel smoothing techniques.

- The trajectory data of the reaction mechanism was generated based on the optimization techniques of genetic algorithm (GA).

- The NPCA-NN algorithm was then used for the reduction of Dimethyl Ether (DME) mechanism.

- An in-house wo-dimensional Computational Fluid Dynamics (CFD) code was developed based on Meshfree technique and time marching solution algonthm.

- The project also provided valuable research experience to two graduate students.

\subsection{Neferences}

1. Haby, M., and Miranda, R, 1999, "Empirical Dynamical System Reduction 1: Global Won-linear Transformations," Center de Recherches Mathematiques, CRM proceedings and Lecture Notes, Vol.20, pp $41-63$.

2. Tomlin, A. S, Turanyi, T, and Pilling, M. J, 1997, Mathematical tools for the construction, investigation and reduction of combustion mechanisms, in Low Temperature Combustion and Autoignition (eds) M. I, Pilling and GHancock, Elsevier, $10.293-437$

3. Jam, S.H, and Goussis, D.A, 1988, Understanding Complex Chemical Kinetics with Computational Singular Perturbation," 22 nd Symposium (international) on Combustion, 10. $931-941$

4. Waas, U, and Pope, S. B, 1992, "Simplifying Chemical Kineticst Intrinsic LowDimensional Manifolds in Composition Space," Combustion and Flame, Vol.88, pp. 239 264.

5. Butuk, NK, and Pemba, JP.200Ib "Computing CHEMKN Sensitivities Using Complex Variables" Preprint, submitted to AS ME Journal of Engineering for Gas Turbines and Power, also presented as ASME Paper No. ETCE2001-17013, ASME Bngineering Technology Conference on Energy, Houston, TX, February 5-7,2001.

6. 3utuk, N.K, and Johnson K, JPGC2001/FACT-19095, Construction of Low Dimensional Reaction State Space Trajectories Using Nonlinear Principal Component Analysis", 2001 International Joint Power Generation Conference, New Orleans, ouisiana, June 4-7,2001.

7. 3harath, R, and Drosen, J., 1994, Neural Network Computing. Windcrest/McGraw-Hill, Vew York:

8. Kramer, M.A, 1991, Non-linear Principal Component Analysis using Autoassociative Veural Networks, AIChE Journal, Yol, 37, No.2, pp 233-243 